
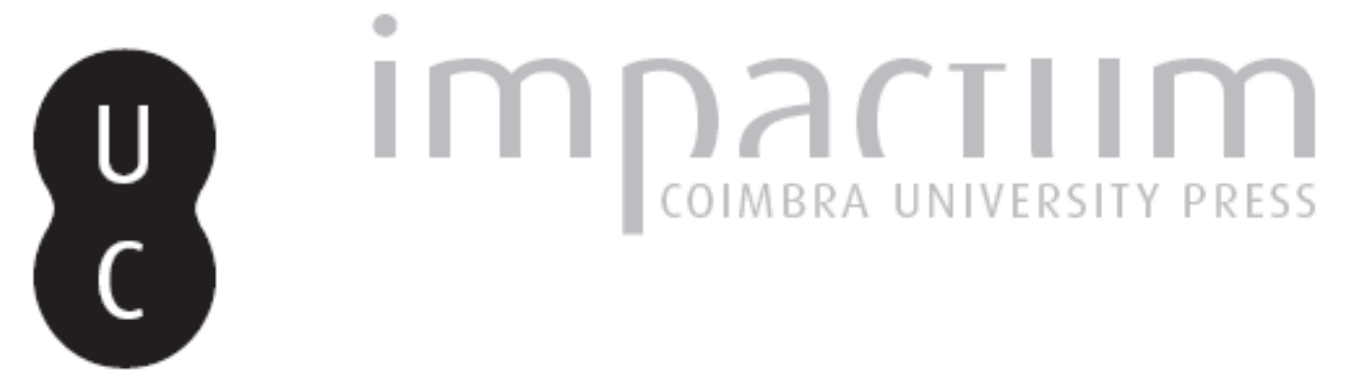

\title{
Sordera por activación del airbag: un caso
}
Autor(es):
Hernando Lorenzo, Antonio E.; Gómez-Guillamón, Ferreol García-Nieto; Moreno Anaya, Pilar; Menchaca Anduaga, Arántzazu

Publicado por: Imprensa da Universidade de Coimbra

URL persistente:

URI:http://hdl.handle.net/10316.2/33265

DOI:

DOI:http://dx.doi.org/10.14195/1647-8630_23_7

Accessed : $\quad$ 26-Apr-2023 11:14:35

A navegação consulta e descarregamento dos títulos inseridos nas Bibliotecas Digitais UC Digitalis, UC Pombalina e UC Impactum, pressupõem a aceitação plena e sem reservas dos Termos e Condições de Uso destas Bibliotecas Digitais, disponíveis em https://digitalis.uc.pt/pt-pt/termos.

Conforme exposto nos referidos Termos e Condições de Uso, o descarregamento de títulos de acesso restrito requer uma licença válida de autorização devendo o utilizador aceder ao(s) documento(s) a partir de um endereço de IP da instituição detentora da supramencionada licença.

Ao utilizador é apenas permitido o descarregamento para uso pessoal, pelo que o emprego do(s) título(s) descarregado(s) para outro fim, designadamente comercial, carece de autorização do respetivo autor ou editor da obra.

Na medida em que todas as obras da UC Digitalis se encontram protegidas pelo Código do Direito de Autor e Direitos Conexos e demais legislação aplicável, toda a cópia, parcial ou total, deste documento, nos casos em que é legalmente admitida, deverá conter ou fazer-se acompanhar por este aviso.

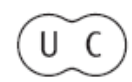


REVISTA PORTUGUESA

$\stackrel{\infty}{\cdots}$

$\stackrel{2}{i}$

$\dot{\bar{x}}$

$\bar{x}_{\bar{x}}$

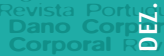

Oeno dorporal Revista Portuguesta do Dano Corporal Revista Portuguens do Dano Corporal

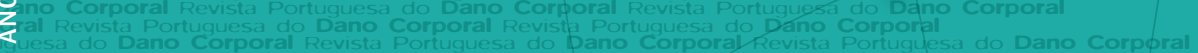

Rvista Portuguesa do Dano Corporal Revista Portuguesa do Dano Corporal Revista Portuguesa do pang Coporal

NPortuguesa do Dano Corporal Revista Portuguesd do Dano Corporal

Silo

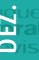

\section{APADAC}

ASSOCIAÇÃO PORTUGUESA

DE AVALIAÇÃO

DO DANO CORPORAL

INSTITUTO NACIONAL

DE MEDICINA LEGAL, I.P.

DELEGAÇÃO DO CENTRO 


\title{
Sordera por activación del airbag: un caso
}

\author{
Antonio E. Hernando Lorenzo(1), Ferreol García-Nieto \\ Gómez-Guillamón(1), Pilar Moreno Anaya(2), Arántzazu Menchaca \\ Anduaga ${ }^{(3)}$
}

Caso: Se trata de una conductora de 71 años, que sufre accidente de tráfico consistente en la colisión de una motocicleta contra el turismo que ella conducía, produciéndole daños en parachoques, rejilla, indicador, proyector, aleta y capó. En el accidente, se habrían activado los airbags lateral izquierdo de puerta del conductor y el airbag de techo, elementos de serie en este vehículo.

La conductora del turismo acudió a Urgencias refiriendo sordera y acúfenos. La exploración física, incluída otoscopia no mostró alteraciones, por lo que se descarta rotura tímpánica o hemotímpano. En las audiometrías realizadas presentó déficit de la agudeza auditiva por pérdida total de la audición en el oído izquierdo y una pérdida de 80 decibelios en el oído derecho y acúfenos. Una otoscopia repetida mostró integridad de las membranas timpánicas. Las pruebas de imagen (TAC) mostraron ausencia de hallazgos patológicos, (no fractura de peñasco del hueso temporal, no luxación de cadena de huesecillos, no presencia de líquido en oído medio o interno, etc.), siendo tratada con corticosteroides, persistiendo la sintomatología, realizándose posteriormente potenciales evocados que mostraron en oído derecho una hipoacusia indeterminada con umbral de $50 \mathrm{~dB}$, y en oído izquierdo hipoacusia neurosensorial con umbral de $60 \mathrm{~dB}$.

\section{AIRBAG Y SEGURIDAD VIAL}

Los airbags son útiles para reducir la gravedad de lesiones en los accidentes de tráfico en los que se ven involucrados automóviles. Basados en todos los

\footnotetext{
1 Médico especialista en Medicina Intensiva; Perito Médico; Master en VDC

Médico especialista en Radiodiagnóstico; Neuroradiólogo

3 Enfermera; Directora de Enfermería del SUMMA 112, Madrid
} 
tipos de accidentes, los airbags reducen las muertes de un 21 a un 22\% para conductores que no utilizan cinturones de seguridad, y de un 9 a un $16 \%$ en conductores que usan cinturón ${ }^{(1,2)}$.

Los airbags son un tipo de sistema de retención en el vehículo que están diseñados para hincharse rápidamente durante una colisión del automóvil, para evitar que los ocupantes golpeen el interior del compartimento del vehículo. Se despliegan a unos 6 litros por milisegundo, y los airbags de los años 90 se hinchaban completamente en aproximadamente 20-30 milisegundos, con una cantidad increíble de fuerza y a una velocidad 157 y $338 \mathrm{~km} / \mathrm{h}$., necesarias para desplegarse lo suficientemente rápido para que su inflado se produzca antes de que el ocupante del vehículo golpee el volante o el salpicadero, durante el desarrollo de un accidente ${ }^{(3)}$.

La intensidad de ruido asociada al despliegue del airbag en un accidente de automóvil, varía con el tipo, tamaño y situación del airbag. El despliegue de un airbag delantero en la posición de conductor producirá niveles medios pico en la presión de sonido de, aproximadamente, 160 dB (Decibelios), en el lado del acompañante de $168 \mathrm{~dB}$, y los dos simultáneamente de $170 \mathrm{~dB}$. El nivel umbral de dolor por ruido es de, aproximadamente, $140 \mathrm{~dB}$, y una simple exposición a esta presión de sonido puede producir pérdida permanente grave de la audición.

El desarrollo más reciente de los airbags laterales aumenta este problema, puesto que genera una presión media pico de sonido de aire de $170 \mathrm{~dB}$, más del 20\% superior al nivel necesario para producir una pérdida de audición permanente grave. Además, al desplegarse más próximo al oído el peligro se ve aumentado.

\section{AIRBAG Y LESIONES OTOLÓGICAS}

El despliegue del airbag ha introducido un nuevo espectro de lesiones, la mayoría relativamente leves y generalmente consisten en eritema, abrasiones y contusiones en cara, zona anterior de cuello o zona alta del tórax. Es infrecuente la producción de lesiones más graves pero se han descrito en la literatura traumatismos con riesgo vital ${ }^{(4)}$, entre otros rotura de aorta ascendente $^{(5)}$. Son de especial importancia las lesiones en ojos, columna cervical, nervio facial, articulación temporo-mandibular, esqueleto facial y vía aérea superior ${ }^{(6,7,8,9,10)}$.

Las lesiones otológicas son poco frecuentes y los trabajos publicados incluyen pocos casos y generalmente se refieren a casos aislados de pérdida 
repentina de audición neurosensorial asociada al inflado del airbag en sujetos generalmente sin patología auditiva previa. Se han referido déficits otológicos tras la activación de airbag, tales como pérdida de audición temporal o permanente, acúfenos, vértigo, otalgia y perforaciones de membrana timpánica como posibles consecuencias ${ }^{(6)}$. Los casos más citados se refieren a pérdida permanente de audición, acompañado en algún caso de acúfenos y alteraciones del equilibrio ${ }^{(7,8)}$.

McFeely ${ }^{(14)}$ refiere 20 pacientes que sufrieron lesiones otológicas como consecuencia del inflado del airbag. Los síntomas más frecuentes fueron pérdida de audición en 17 (85\%) y acúfenos en 17 (85\%). Diez pacientes (50\%) tenían trastornos del equilibrio y cuatro (20\%) tenían perforación de membrana timpánica. La orientación hacia el airbag se vio que se asociaba a la pérdida de audición, a los pródromos de jaqueca, (aural fullness) y a perforación de membrana timpánica. De los 17 pacientes con pérdida subjetiva de audición, fue unilateral en 10 (59\%), bilateral en 7 (41\%) y persistente en $15(76 \%)$. La pérdida objetiva de audición (caída de al menos $10 \mathrm{~dB}$ en dos frecuencias) se documentó en 21 de 24 (88\%) oídos afectados subjetivamente; esto incluyó pérdidas de audición unilaterales y bilaterales neurosensitivas, unilateral de conducción y mixta. Fue neurosensorial en 17 (81\%), de conducción en $2(10 \%)$ y mixta en $2(10 \%)$. Se afectaron más frecuentemente las altas frecuencias $(>4 \mathrm{kHz})$, seguido de frecuencias medias $(2$ a $4 \mathrm{kHz}) \mathrm{y}$ bajas frecuencias $(<2 \mathrm{kHz})^{(9)}$.

La pérdida de audición traumática tras inflado de airbag es un incidente raro o excepcional, que probablemente se produce en sujetos con una susceptibilidad y sensibilidad particulares ${ }^{(10)}$. De 71 pacientes referidos con síntomas otológicos después del despliegue del airbag(11) ${ }^{(1)}$ seleccionaron a 22 pacientes para evaluar el riesgo individual. Se confirmó un daño otológico transitorio debido al despliegue del airbag, con una elevación temporal en el umbral acústico. La persistencia de un déficit auditivo puede ser debida a cofactores a través de un mecanismo metabólico, angiopático, neuropático o desconocido, ya que los 6 pacientes sin daño otológico presentaban analítica normal, mientras que 12 de los 16 pacientes con daño otológico tenían niveles alterados de glucosa, urea y colesterol en sangre, con diferencias estadísticamente significativas en comparación con los sujetos normales. La edad de los pacientes también podría afectar de una forma significativa la recuperación del trauma acústico ${ }^{(12)}$.

En un estudio en la Universidad de Michigan, de 177 ocupantes de automóviles que habían sufrido accidentes con despliegue de airbag, tanto de 
volante solo como también de ocupante, solo 3 refirieron tras el accidente algún tipo de problemas relacionados con la audición tras el accidente $(1,7 \%)^{(13)}$.

Como mencionamos al principio, la activación de los airbags se asocia a un riesgo inherente, que incluye ruido de alta amplitud y corta duración por el despliegue del airbag.

Una revisión de la investigación disponible en la industria del automóvil, indica que el pico de amplitud de este ruido puede superar los $170 \mathrm{~dB}$ de nivel de presión de sonido.

Los airbags de automóviles se despliegan por la generación rápida de un gran volumen de gas. Esto crea una onda de presión breve (menor de 100 milisegundos), e intensa (150 a $170 \mathrm{~dB}$ ) que se propaga a través del compartimento de pasajeros. La onda de presión de sonido producida se relaciona con factores tales como el tamaño del vehículo, el número de ocupantes y la ventilación.

Richard Price presenta 70 pacientes con síntomas otológicos tras la activación del airbag en el automóvil que ocupaban. De ellos, a 61 se les realizó audiograma tras el despliegue del airbag; de éstos, 50 mostraban pérdida de audición en una o más frecuencias en el oído con molestias otológicas.

Describe el impulso de ruido como un brote de sonido breve (como la explosión de un disparo), en oposición al ruido contínuo, tal como el de una lavadora, y que eventos menores tales como golpear con un martillo sobre un clavo, puede producir pérdida de audición. También concluyó que, en oposición a lo pensado hasta entonces, cuando se tienen las ventanas subidas mientras se produce el despliegue del airbag, es menos peligroso para el oído que con las ventanas bajadas. Ello se debe a que la presión más alta generada en la cabina cerrada aumenta la rigidez del huesecillo estribo, en el oído medio, limitando la transmisión de energía al oído interno y evitando mayor daño al oído. Asimismo, el mayor volumen de los airbags de los automóviles norteamericanos respecto a los automóviles europeos, podría suponer un riesgo adicional de mayor lesión auditiva.

El estudio se hizo utilizando el Algoritmo de Valoración de Riesgo Auditivo para Humanos (AHAAH), modelo que utiliza componentes anatómicos de la estructura del oído, para predecir la pérdida de audición debida a exposición a sonidos intensos por encima de $130 \mathrm{~dB}$. Se ha visto que predice la pérdida de audición de manera exacta en el 95\% de casos en los cuales se ha visto expuesto el oído humano. 
En el estudio de Price, los resultados indicaban que el 17\% de las personas expuestas a la conmoción por sonido por el despliegue de un airbag en un accidente de automóvil, sufría pérdida permanente de la audición.

El daño consiste en rotura timpánica, luxación de los delicados huesos del oído medio y daño importante al oído interno. Eso puede producir pérdida de audición, acúfenos (zumbidos u otro tipo de sonidos en los oídos) y vértigo (sensación de inestabilidad y mareo) $)^{(14)}$.

Aunque la afectación auditiva con pérdida por trastorno neurosensitivo o neurosensorial en niños es muy infrecuente, se ha referido el caso de una pérdida auditiva neurosensorial significativa tras el despliegue del airbag en un paciente pediátrico(15).

En una serie de 20 casos, los síntomas más frecuentes fueron pérdida de audición, (neurosensorial, de conducción o mixta) y acúfenos en el 85\% de pacientes; sin embargo el 50\% tenían desequilibrio, 20\% tenían perforación de membrana timpánica, 30\% se quejaban de sensación de plenitud aural y 15\% sufrían dolor crónico relacionado a disfunción de la articulación temporomandibular. La orientación del oído hacia el airbag se vio que se asociaba a pérdida de audición, plenitud aural o aura de cefalea, y perforación de tímpano.

Los acúfenos (percepción de sonido en ausencia de una estimulación sonora externa) pueden deberse a anormalidades en la cóclea, nervio coclear, vías auditivas ascendentes o corteza auditiva, siendo una de las molestias residuales más frecuentes tras la activación de airbag.

En el Primer Encuentro Panamericano-Ibérico sobre Acústica del año 2002 se revisan 66 pacientes con edades entre 4 a 81 años y encuentran acúfenos permanentes del 52 al 79\%, pérdida de audición del 47 al 71\%, vértigo (incluyendo vértigo posicional paroxístico benigno) del 20 al 30\%, perforación de membrana timpánica del 6 ó $9 \%$ y fístula perilinfática el 1 ó $2 \%$.

La fístula perilinfática es una rotura en la membrana oval y/o redonda que deja escapar líquido en el oído medio. Es de presentación rara pero puede producirse durante levantamiento de pesos, traumatismos craneales y buceo en aguas profundas.

También se han descrito por trauma acústico el ruido agudo e intenso de una sirena de coche de bomberos, por efecto de un rayo y por complicaciones por implantes cocleares y cirugía del hueso estribo. 
Durante los despegues y aterrizajes de un avión se pueden producir problemas en la igualación de la presión en el oído medio por un resfriado. Los síntomas de una fístula incluyen pérdida de audición, acúfenos, hiperacusia y sensibilidad a los cambios de presión.

También respecto a la incidencia de vértigo y trastornos auditivos tras despliegue de airbag, las lesiones parecen deberse a una combinación de sobrecarga de ruido y fuerzas ocasionales (explosivas) sobre el tímpano. Este mecanismo de lesión también puede ser causa de fístula perilinfática, como se acaba de comentar.

Respecto a la lesión "por explosión", Cunningham y cols. (1999), presentaron un caso aislado de un individuo cuyo oído medio izquierdo fue deshecho por el despliegue de un airbag. Los osículos del oído medio se desplazaron hacia el oído interno, produciendo una sordera total y parálisis facial. En este caso la cabeza del paciente estaba girada hacia la derecha y así el airbag golpeó el oído izquierdo ${ }^{(22)}$. En un estudio más grande, se correlacionaba el tener el oído vuelto hacia el airbag en el momento del inflado, con aura de cefalea y perforación de membrana timpánica ${ }^{(14)}$.

Hipermovilidad del estribo -estapedio-: Según Saunders y cols., la onda portadora de baja frecuencia durante el despliegue del airbag, produce un desplazamiento máximo del estribo y el ligamento anular. Este mecanismo podría causar desgarro del ligamento anular e hipermovilidad del estribo. La hipermovilidad del estribo puede llevar a un vértigo inducido por sonido así como a hiperacusia ${ }^{(23)}$.

Respecto al diagnóstico, se sugiere por el comienzo inmediato de síntomas de audición tras el despliegue del airbag en ausencia de síntomas preexistentes, recomendándose audiometría normal y de alta frecuencia, test de emisiones, timpanometría y pruebas de acúfenos (si se precisan).

La edad avanzada fue una variable significativamente estadística asociada a la pérdida de audición en el estudio de McFeely y $\operatorname{cols}^{(14)}$.

Respecto a la perforación de membrana timpánica inducida por airbag, se cree que el umbral para la perforación de una membrana timpánica normal está alrededor de $180 \mathrm{~dB}$, pero puede ser inferior, en $160 \mathrm{~dB}$, con enfermedades otológicas preexistentes. En un grupo de 4 sujetos con perforaciones, ninguno mostró curación espontánea, precisando intervención. Esto está en contraste con las situaciones de combate, en las cuales las tasas de resolución varían 
de un $81 \%$ a un $91 \%$. Las perforaciones de mayor tamaño y las localizadas posteriormente y las que no muestran curación en pocas semanas, tienen una tasa significativamente menor de cierre.

El estudio de McFeely y cols. ${ }^{(14)}$ sugiere que la posición del oído hacia el airbag fue un factor importante en relación a la pérdida de audición, perforación de membrana timpánica y pródromos de jaqueca (aural fullness). Un oído que esté dirigido hacia la onda explosiva (blast), experimenta una presión en la membrana timpánica que es aproximadamente el doble de la de un oído no enfrentado a la onda.

Los pacientes con perforación de membrana timpánica en este estudio tenían sus oídos enfrentados hacia el airbag que se inflaba directamente delante de ellos; en base a los resultados de este estudio, la introducción de airbags laterales en los nuevos vehículos, puede aumentar las posibilidades de perforación de membrana timpánica y de otras lesiones otológicas. Factores adiciones que se considera podrían ser importantes con respecto a la perforación de membrana timpánica, son el tamaño y la geometría interior del vehículo, la situación del ocupante y el número de airbags desplegados.

Aunque se pensaría que un volumen interior efectivo más pequeño y las ventanas cerradas aumentarían la posibilidad de trauma acústico, esto no es así, porque no toma en consideración el efecto protector del componente de ruido de inflado de baja frecuencia, el cual reduce la efectividad del componente de alta frecuencia para producir una desviación de umbral (TS) en voluntarios humanos y puede modular el flujo de energía en la cóclea.

Sorprendentemente el tener las ventanas abiertas podría ser menos protector, en base a modelos matemáticos. La diferente geometría interior del vehículo también se sabe que afecta al ambiente de presión y puede contribuir a lesión otológica. También el despliegue de múltiples airbags, lógicamente aumenta la producción de ruido.

Buckley G., Setchfield N. Frampton R. ${ }^{(16)}$, refieren el caso de dos mujeres que tras activación de airbag en accidente de tráfico a baja velocidad, presentan una pérdida de audición neurosensorial bilateral a bajas frecuencias, con acúfenos y pérdida de audición desde el momento del accidente, produciéndose la recuperación parcial de audición en un oído en la primera de ellas.

Ohki M. y cols, describen recientemente (2012) otro caso de sordera neurosensorial en un ocupante de un automóvil en Japón, quien sufrió una 
sordera en oído derecho tras activación del airbag, con recuperación parcial de la audición tras tratamiento con corticoides ${ }^{(24)}$.

\section{REFERENCIAS}

1 Zador PL, Coccone MA. "Automobile driver fatalities in frontal impacts: air bags compared with manual belts". Am J Pub Health 1993; 83:661-6.

2 O'Neill B, Lund AK. “The effectiveness of air bags in preventing driver fatalities in the united states". Proceedings of the International Conference on Air Bags and Seat Belts: Evaluation and Implications for Public Policy, Montreal, Canada, October 18-20, 1992).

3 Nacional Highway Traffic Safety Administration, "Air Bag Deployment Characteristics", Springfield, VI, Nacional Technical Information Service, 1992.

4 Lund AK, Ferguson SA. "Driver fatalities in 1985-1993 cars with airbags", J Trauma 1995; 38(4):469-475).

5 de Guzman B.J., Morgan A. S., “Aortic Transection Following Airbag Deployment", NEJM., Vol. 337, No. 8, Aug. 21, 1997, pág. 573-574),

6 Klask J. ("Injuries in the throat-nose-ear area by automobile air bags", Laryngorhinootologie. 2001 Mar; 80(3):146-51).

7 Saunders JE, Slattery WH 3rd., Luxford WM (“Automobile airbag impulse noise: otologic symptoms in six patients”, Otolaryngol Head Neck Surg. 1998 Feb; 188(2):228-34).

8 Stankiewicz C., Przewozny T., y Kizlowski J. ("Noise from a car airbag as a cause of acute acoustic trauma". Otolaryngol Pol. 2000; 54(6):775-81).

9 McFeely WJ, Bojrab DI y cols., "Otologic injuries caused by airbag deployment", Otolaryngology, Head and Neck Surgery, Vol. 121, No. 4, Oct 1999, pág 367-373.

10 Bonelli A., Fontanella WM y cols. (“Airbag and hearing loss: our experience.", Rev Laryngol Otol Rhinol (Bord). 2002; 123(1):53-4).

11 Yaremchuk D., Dobie RA. (“Otologic injuries from airbag deployment”, Otolaryngol Head Neck Surg. 2001 Sep; 125(3):130-a).

12 Passáli D, Passáli GC, Passáli FM y cols. ("Airgabs and permanent auditory deficits. A real correlation?”. Acta Otorhinolaryngol Belg. 2003; 57(3):177-81).

13 Huelke DF, Moore JL y cols. ("Hearing loss and automobile airbag deployments". Accid. Anal Prev. 1999 Nov; 31(6):789-92).

14 Price R. "Intense impulse noise: hearing conservation's poison gas". Paper presented at: Annual Conference of the National Hearing Conservation Association, February 16, 2007.

15 Chao N., Pomerantz WJ ("Illustrative Cases: Acute Hearing Loss After Airbag Deployment”. Pediatric Emergency Care: October 2004 - Vol. 20 - No. 10 - pp 683-686).

16 Buckley G., Setchfield N. Frampton R., "Two case reports of possible noise trauma after inflation of air bags in low speed car crashes", BMJ, Vol. 318, 20 Feb. 1999, 499-500.

22 C. D. Cunningham, P. C. Weber, and J. Cur'e, "Neurotologic complications associated with deployment of airbags," Otolaryngology, vol. 123, no. 5, pp. 637-639, 2000. 
23 Saunders, J. E., W. H. Slattery, 3rd, et al. (1998). "Automobile airbag impulse noise: otologic symptoms in six patients." Otolaryngol Head Neck Surg 118(2): 228-234.

24 Ohki M., Ishikawa J., Tahara A, "Case Report: Sensorineural hearing Loss due to Air Bag deployment”, Case reports in Otolaryngology, Vol 2012, Article ID 203714, 2 pags.

Resumo: Surdez por ativação de airbag: um caso

Descreve-se o caso de uma condutora de 71 anos que sofreu um acidente por embate lateral de um motociclo no lado esquerdo do automóvel que conduzia. 0 airbag lateral e 0 do teto foram ativados. Não tinha antecedentes patológicos dignos de registo. Foi ao hospital por perda de audição e zumbidos. A otoscopia era normal. A audiometria mostrou hipoacusia completa no ouvido esquerdo e perda de $80 \mathrm{~dB}$ no ouvido direito, tendo sido medicada com corticoesteroides. Porque persistia a perda de audição efetuou-se TAC do ouvido, que não mostrou alterações nem lesão dos ossículos. Os potenciais evocados mostraram no ouvido direito uma hipoacusia indeterminada com o limiar de $50 \mathrm{~dB}$ e no ouvido esquerdo hipoacusia neurosensorial com o limiar de $60 \mathrm{~dB}$. Os autores fazem uma revisão dos mecanismos da surdez provocada por airbag.

Palavras-Chave: Surdez; hipoacusia; airbag; audiometria; potenciais evocados.

Summary: Deafness by activation of the airbag: a case

A 71 year old female car driver with no previous illness was involved in a lateral collision in which a motorcycle collided front-end on the left side of the car she was driving. Left door side airbag and left curtain airbag were activated. She presented to hospital with auditory loss and tinnitus. Otoscopy exam was normal. Audiometry showed complete loss of hearing in left ear and $80 \mathrm{~dB}$ loss in right ear. She was treated with corticosteroids. As loss of hearing persisted, CT scan was performed, not showing abnormalities nor injury to the ossicle chain; sensoryneural evoked potentials showed undetermined right hypoacusia with $50 \mathrm{~dB}$ threshold and left sensoryneural hearing loss with $60 \mathrm{~dB}$ threshold. Deafness mechanisms produced by airbag are reviewed.

Key-Words: Deafness; hypoacusia; airbag; audiometry; evoked potentials.

Pedido de separatas:

ANTONIO HERNANDO LORENZO

herloren@telefonica.net 